\title{
Onset of subharmonics generated by forward wave interactions in $\mathrm{Bi}_{12} \mathrm{SiO}_{20}$
}

\author{
D. J. Webb, L. B. Au, D. C. Jones, and L. Solymar \\ Holography Group, Department of Engineering Science, University of Oxford, Parks Road. \\ Oxford OX1 3PJ, Unised Kingdom
}

(Received 22 February 1990; accepted for pubication 30 July 1990)

\begin{abstract}
The threshold conditions, under which a spatial subharmonic beam may arise when a $\mathrm{Bi}_{12} \mathrm{SiO}_{20}$ crystal is illuminated by two pump beams, are investigated. It is shown that a noninear theory based on the material equations leads to good qualitative agreement with experiments
\end{abstract}

Two-wave mixing phenomena in photorefractive media may be usually described using the model first proposed by Kukhtarev et al. In this model, the variation of the amplitude of the incident light beams due to the presence of a refractive index modulation is determined by the so-called field equations, while the formation of the index modulation in response to the intensity standing wave pattern is governed by the material equations. Recently, experiments with $\mathrm{Bi}_{12} \mathrm{SiO}_{20}$ (BSO) have shown that, under the appropriate conditions, one or more spatially subharmoric beams may arise between the pump beams at angles that are simple fractions of the interpump angle. ${ }^{3}$ In a previous publication, ${ }^{4}$ these subhamonic beams have been characterized as a function of detuming and applied electric field, but as yet the mechanism responsible for the origin of these subharmonics has not been identified to any degree of certainty.

It has been proposed by Ringhofer and Solymar that the mechanism responsible for the generation of subharmonics is selective amplification of a signal scattered in the right direction. In the present letter we suggest another mechanism based on the material equations and check experimentally the predictions of the model for the onset of the second subharmonic the one appearing haifway between the two pump beams).

The basic experimental setup is shown in Fig. 1. A spatially filtered, expanded and collimated beam of light from an $\mathrm{Ar}^{+}$laser, emitting at $514.5 \mathrm{~nm}$, was split in two. A small frequency shift was applied to one of the beams by reflecting the beam from a mirror mounted on a $\mathrm{PZ}$ driven by a serrodyne waveform. The two beams were then directed at the 110 face of a crystal of $\mathrm{BSO}$. This face measured $6 \times 7 \mathrm{~mm}^{2}$ and the length of the crystal was $10 \mathrm{~mm}$. In addition, collimated light from a $\mathrm{Kr}^{+}$laser emitting at $530.9 \mathrm{~nm}$ was also incident on the crystal. This light and the ight from the $\mathrm{Ar}^{+}$laser were mutually incoherent, and hence the intensity of the light from the $\mathrm{Kr}^{-+}$laser could be varied in order to adjust the modulation of the intensity standing-wave pattern produced by the pump beams.

The ratio of the intensities of the two beams from the Ar laser was 1.2:1, and the total incident power density was maintained at $5 \mathrm{~mW} / \mathrm{cm}^{2}$. The beams were polarized perpendicular to the 001 face and the electric field was applied perpendicular to the 110 face of the crystal. The pump beams were angled so as to write a grating within the BSO with a period of $20 \mu \mathrm{m}$. The intensity of the subharmonic was measured by focusing the subharmonic beam onto a power meter using a $50 \mathrm{~cm}$ focal length convex lens.

With the $\mathrm{Kr}^{+}$laser initially switched off, the subharmonic bearn intensity was recorded as a function of detuning frequency at a number of different electric fields. In this way, for each value of the appied electric field, the optmum intensity was obtained. This process was repeated with the $\mathrm{Kr}^{+}$laser contributing different amounts of power to the total incident intensity, and therefore varying the modulation. The total range was from 0.37 to 1 for modulation and from 1.6 to $10 \mathrm{kV} / \mathrm{cm}$ in applied electric feld. We found that the subharmonic power was always higher as either the modulation or the applied electric field increased. The maximum power in the subharmonic was $15 \mu \mathrm{W}$ for a modulation of 1 and an electric field of 10 $\mathrm{kV} / \mathrm{cm}$. The aim of this letter is to study the threshold, i.e., our interest is in the values of modulation and electric field which, at optimum detuning, cause the appearance of a subharmonic beam. There is bound to be some uncertainty concerning the measurement of these threshold values for the reason that there is always some light scattered in the direction of the subharmonic beam even in the absence of an applied electric field. Our criterion for the threshold is that the newly appearing subharmonic intensity just equals the scattered light intensity, and any small increase in electric field or modulation will cause a large increase in subharmonic power. The threshold values measured according

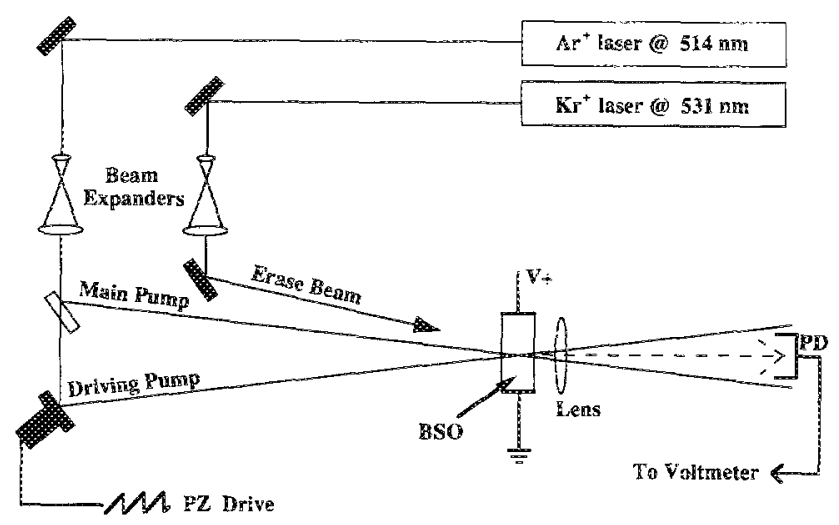

FIG. I. Fxperimental arrangement used to find threshold feld for the appearance of subharmonics at a given modulation. 


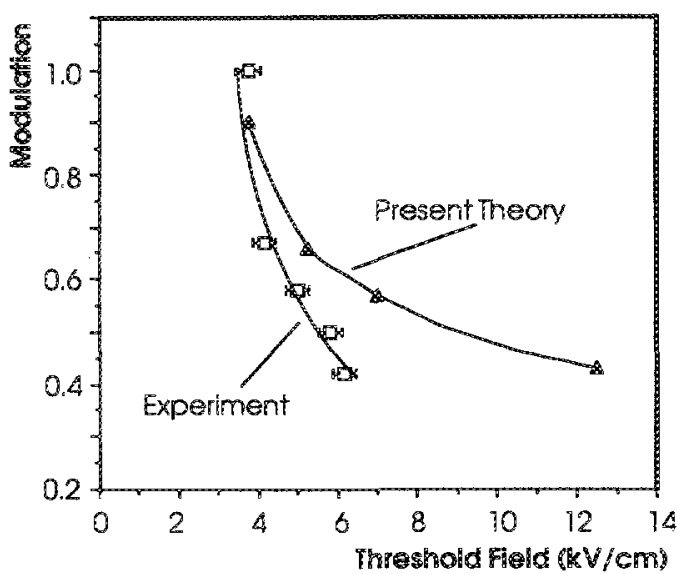

FIG. 2. Plot of threshold field for the appearance of subhamonics against modulation. (Open squares) experimental results; (filled triangles) theoretical resuits.

to this defnition are plotted in Fig. 2. It may be seen that lower modulation will suffice for the generation of subharmonics as the electric field increases.

The aralysis is based on the theoretical papers of $\mathrm{A} u$ and Solymar ${ }^{6,7}$ in which the nonlinear material equations are solved by a numerical method yielding the Fourier components of the electron density, ionized space-charge density, and space-charge field.

We must assume in the calculation that there is an input modulation both at the fundamental component $m_{1}$ and at the subhamonic component $m_{1 / 2}$. For most values of the parameters, it is found that the fundamental components have negligible infuence upon the subharmonic components. But at certain values of $m_{1}$ when the applied electric field increases we find that, for a smal increase of the applied electric field there is a large increase in the subharmonic space-charge field. Taking a concrete case, we find for example that for a certain set of materials param. eters (given below) and for a fundamental modulation of $m_{1}=0.9$ and applied field $=3.5 \mathrm{kV} / \mathrm{cm}$, the subharmonic space-charge field is $3.3 \times 10^{-4} \mathrm{kV} / \mathrm{cm}$, whereas for an electric feld of $4 \mathrm{kV} / \mathrm{cm}$ the subharmonic space-charge feld increases to $1.5 \mathrm{kV} / \mathrm{cm}$, an increase by a factor of 5000 . This is a clear indication of a sudden onset of interaction between the fundamental and the subhamonic conponent of the space-charge field. We claim then that the threshold applied electric field is $4 \mathrm{kV} / \mathrm{cm}$.

The rumerical calculations are rather laborious to perform. In order to calculate accurately the subharmonic component of the space-charge feld as many as 26 higher harmonics are required in the Fourier expansion. It can take as long as 4 h of CPU time on our DEC VAX 1/780 computer to obtain the maximum value of the spacecharge field for a given electric field.

Figure 2 shows the relationship between the threshold electric field and $m_{1}$, using the following crystal parameters: lonized acceptor density, $10^{22} \mathrm{~m}^{-3}$; refractive index, 2.62 ; electro-optic constant, $3.4 \times 10^{-12} \mathrm{~m} / \mathrm{V}$; temperature, $293 \mathrm{~K}$; recombination coefficient, $1.6 \times 10^{-17} \mathrm{~m}^{3 /} / \mathrm{s}$; electron mobility, $10^{-5} \mathrm{~m}^{2} / \mathrm{V}$; photoionization corstant,
$2 \times 10^{-5} \mathrm{~m}^{2} / 5 ;$ donor density, $10^{25} \mathrm{~m}^{-3}$. The total incident power density was $5 \mathrm{~mW} / \mathrm{cm}^{2}$, the wavelength 514.5 $\mathrm{nm}$, and the grating spacing $20 \mu \mathrm{m}$. Examination of Fig. 2 shows that the theory is in good qualitative agreement with the experimentai results.

We believe that there are two distinct mechanisms responsibie for the emergence of subhamonics. One is based on the field equations concerned with the coupling of the waves as described in Ref. 5. The other one is based upon the materials equations, the subject of the present letter. In practice, both mechanisms are bound to be present. The latter one is responsible for the sudden rise of the subharmonic and the former one causes its further amplification. When we consider the threshold conditions only then, we believe, the materials equations will suffice, and this seems to be confirmed by the good qualitative agreenent between theory and experiment.

As mentioned before, the numerical calculations are rather demanding so the chances seem to be rather low that the theory, as it stands, could accurately predict the subhamonic output. We can, nevertheless, indicate by a particuar example the mechanism by which we belicve the subharmonic arises.

Let us assume that at the input of the crystal we have some scattering in the drection of the subharmonic beam. We take the scattered power as $5 \times 10^{-13}$ times smaller than the input power leading to a modulation (between the subharmonic beam and one of the pump beams) of $10^{-6}$. Let us further assume that the conditions are right for the onset of subharmonic interaction, the applied electric field being $4 \mathrm{kV}$ and the parameters as given previcusly. Then, due to the interaction between the fundamental grating and the subharmonic grating, we have an enomous intensity gain coefficient, calculated from the space-charge ficld as $\Gamma=6729 / \mathrm{cm}$. Assuming that this gain coefficient remains constant for a distance of $6.8 \mathrm{~mm}$, the subharmonic power increases by a factor of 100 and the subharmonic modulathon increases by a factor or 10 to $m_{1 / 2}=10^{-5}$. It is a very important characteristic of the interaction mechanism discussed that the interaction decreases as the intensity of the subharmonic component increases. For $m \frac{1 / 2}{=2} 10^{-5}$, the gain coefficient may be calculated as $\Gamma=2128 / \mathrm{cm}$. We reed now a distance of $21.6 \mathrm{hm}$ for the modulation to increase to $m_{1 / 2}=10^{-4}$. The corresponding gain coeffcient is now $\Gamma=673 / \mathrm{cm}$ and a distance of $68.4 \mu \mathrm{m}$ is needed for a further increase in modulation to $m_{1 / 2}=10{ }^{3}$. Thus, in a distance of less than a tenth of a mm, we predict an amplification of the subharmonic beam of six orders of magnitude. As $m_{1 / 2}$ rises further the fundamental-subharmonic interaction disappears, and we are left eventualy by the usual gain coefficient which in out present case amounts to about $20 / \mathrm{cm}$.

As it turns out the results of the calculation are sensitive to the choice of the parameters particularly to the value of mobility. Considering further the large number of parameters, the uncertainty about their exact value, and the complexity of the numerical calculations we feel that further experimental work needs to be done which night give clues for simplifying the theoretical approach. Assum- 
ing that the crystal would produce a subharmonic, the ideal experiment that could shed a lot more light upon the mechanism is as follows. Measure both the threshold conditions and the subharmonic output as a function of detuning for a set of BSO crystals grown under identical conditions and having thicknesses of (say) $1 \mathrm{~mm}, 2 \mathrm{~mm}, 3$ $\mathrm{mm}, 5 \mathrm{~mm}$, and $10 \mathrm{~mm}$. If our hypothesis is correct, that is, the threshold conditions are determined by the materials equations only, then these threshold values will be more or less the same for each member of the set. The maximum subharmonic output would, on the other hand, be strongly dependent on the thickness of the crystal since the ficld equations predict an exponential increase with thickness.
The financial support of the U.K. Science and Engineering Research Council is gratefully acknowledged. In addition, L.B.A. wishes to thank the Croucher Foundation of Hong Kong for its generous financial support.

${ }^{1}$ N. V. Kukhtarev, V. B. Markov, S. G. Odulov, M. S. Soskin, and V. L. Vinetskii, Ferroelectrics 22, 949 (1979).

${ }^{2}$ N. V. Kukhtarev, V. B. Markov, S. G. Odulov, M. S. Soskin, and V. L. Vinetskii, Ferroelectrics 22, 961 (1979).

'S. Mallick, B. Imbert, H. Ducoller, J. P. Herriau, and J. P. Huignard, J. Appl. Phys. 63, 5660 (1988).

${ }^{4}$ D. $J$. Webb and I. Solymar, Opt. Commun. 74, 386 (1990).

5 K. I. Ringhofer and L. Solymar, Appl. Phys. B 48, 395 (1989).

${ }^{6}$ L. B. Au and L. Solymar, Opt. Lett. 13, 660 (1989).

${ }^{7}$ L. B. Au and L. Solymar, J. Opt. Soc. Am. A 7, 1554 (1990). 